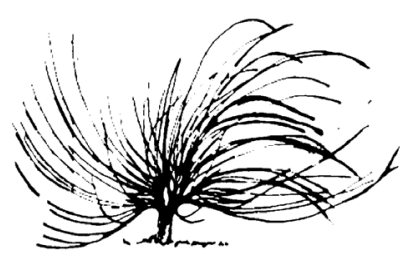

\title{
¿Hay una disminución en la cantidad de vocabulario de los ingresantes a carreras de nivel superior? Análisis longitudinal
}

\author{
Martín Gonzalo Zapico ${ }^{1}$ \\ Instituto de Formación Docente Contiene \\ Universidad Nacional de San Luis \\ Argentina \\ athenspierre@gmail.com \\ Lucía Degregori ${ }^{2}$ \\ Universidad Nacional de Mar Del Plata \\ Argentina \\ lucia.degregori@gmail.com \\ Itatí Berardo ${ }^{3}$ \\ Universidad Nacional de Mar Del Plata \\ Argentina \\ itaa.blue@gmail.com
}

\section{Resumen}

El sistema educativo argentino vive una situación que podría ser catalogada como crisis, que se hace manifiesta cuando los egresados del secundario deben afrontar una carrera de nivel superior o universitaria y no tienen las

\section{(ब⿻肀}

Recibido: 15 de febrero de 2019. Aprobado: 12 de setiembre de 2019.

http://dx.doi.org/10.15359/rep.14-2.9

1 Es profesor de Letras y Filosofía recibido en la UNMDP. Actualmente, se desempeña como Profesor Responsable en el IFDC-San Luis y Doctorando en Educación en la UNSL. Ha realizado numerosas publicaciones en revistas científicas, así como tres libros y asistido a numerosas reuniones científicas en los campos de educación, lingüística y psicología

2 Es profesora de Inglés recibida en la UNMDP.

3 Es profesora de Inglés recibida en la UNMDP. 
competencias básicas de lectura y escritura (entre otras), lo cual redunda en una alta tasa de deserción en el primer año. A su vez, hay ideas que afirman una disminución en estas competencias a lo largo que pasan los años y las nuevas cohortes. En este artículo, pondremos a prueba dicha hipótesis, para lo cual se compararán las puntuaciones obtenidas en un test de vocabulario a cuatro cohortes de ingresantes, y se observarán los cambios en las puntuaciones. Los resultados sugieren una disminución lenta, pero constante en las medias de los resultados, aunque no se observa una diferencia estadísticamente significativa entre las cohortes y las puntuaciones.

Palabras clave: educación, abandono, enseñanza, vocabulario, educación superior.

\begin{abstract}
In the Argentine education system today, we live a crisis situation, which becomes evident when high school graduates must face a college degree: they do not have the basic skills of reading and writing, which results in a high dropout rate in their freshman year. In turn, there are ideas that affirm a decrease in these competences throughout the years and the new cohorts. In this article, we will test this hypothesis. Hence, the scores obtained in a vocabulary test will be compared to four cohorts of new students, and the changes in the scores will be observed. The results suggest a slow but constant decrease in the means of the results, although there is no statistically significant difference between the cohorts and the scores.
\end{abstract}

Keywords: education, drop out, teaching, vocabulary, higher-education

\title{
1. El problema del vocabulario
}

La temática del vocabulario y su vinculación con el desempeño académico ha sido trabajada ampliamente. Se han recabado enfoques puramente cuantitativos (Pavez et al, 2016; Zapico, 2016; Zapico, 2017; Zapico y Delsouc, 2017; Zapico y Zapico, 2018; Zapico 2018), en los cuales se analizó la correlación entre las variables vocabulario y 
abandono, así como la incidencia de un alto vocabulario en las posibilidades de tener éxito a la hora de resolver evaluaciones, entre otras cosas; y se definió la importancia del léxico en el primer año de una carrera de nivel superior. También, se han revisado enfoques más cualitativos sobre el vocabulario (Hirsch, 2004; Bojorque, Bojorque y Dávalos, 2016; Roldán y Zavaleta, 2016; Londoño-Vásquez y Bermúdez, 2018), a través de encuestas de autopercepción, entrevistas y estudios de caso, que establecieron una alta importancia en cuanto a la posibilidad de emplear un vocabulario amplio y el desempeño académico satisfactorio, que a su vez inciden en una alta motivación a la hora de afrontar una carrera. Asimismo, se revisaron ensayos (Álvarez y Martínez, 2011; Zapico, 2017b; Ontibón, 2018) en los cuales se abordaron problemáticas más amplias como la lectura en el nivel superior, la perspectiva transversal del vocabulario y las competencias de lecto-escritura, para acceder a los textos universitarios, la enseñanza del vocabulario en distintos niveles del sistema educativo, entre muchos otros asuntos.

En todos estos textos hay un común acuerdo en cuanto al papel fundamental que el vocabulario adquiere a la hora de cursar una carrera de nivel superior, y en su importancia para la competencia transversal de lectura comprensiva que suele ser estructurante de la trayectoria de un alumno en dicho nivel.

Este estado de situación puede ser documentado de diversas formas: la charla con colegas de distintas instituciones, estudios que revelan las profundas deficiencias en prácticas sencillas como la lectura de un texto y la expresión escrita (Valeiras, 2009; Laco, Natalia y Ávila, 2010), la enorme proliferación de talleres de escritura en los cursos de ingreso a un sinfin de carreras de nivel superior (Carlino, 2001; Carlino, 2007; Moyano, 2005), entre otras. Esta problemática, lejos de pertenecer al plano de lo estrictamente académico, llega también a lo social. Un conocido estudio del presidente de la Real Academia Española Pedro Barcia, llevado a cabo en el año 2007, señalaba que en Argentina los jóvenes usan una cantidad promedio de no más de 240 palabras para comunicarse. Hoy, pasados diez años, se observan las consecuencias de dicho estado de situación, y el asunto parece no mejorar. Incluso los autores más críticos con esa cifra (que señalan que no se tiene en cuenta los vocablos creados por las subculturas juveniles y realizan la llamada "crítica a la cantidad") hablan de una innegable "crisis educativa" en materia del lenguaje (Requejo, 2001; Garfias, 2010). 
Otro indicio que parece apoyar lo planteado en el párrafo anterior son los estudios sobre lectura en jóvenes. Muchos de ellos, realizados tanto en Argentina (Horacek, Ottaviano y Palacios, 2015; Paredes, 2015), Latinoamérica (Hernández García y López, 2015; Amiama-Espailat y Mayor-Ruiz, 2017), Norteamérica (León, 2015; Mumper y Gerrig, 2017) y Europa (Sánchez Lozano, 2003; Martínez, 2017), evidencian que la lectura, ya sea en soporte papel o digital está en un proceso de disminución preocupante. Si bien hay autores que aducen que los textos de las redes sociales son una forma de lectura, desconocen dos críticas fundamentales. Por un lado, oftalmólogos e investigadores de medicina han señalado hasta el hartazgo (Belaunde, Carrillo, Amo y Ayora, 2016; Ledo, 2016; Armengol, Castellanos, Molina, León y Díaz, 2017; Díaz, Reyes y Rangel, 2017; entre muchísimos otros) las consecuencias que la exposición prolongada a la luz de las pantallas de celulares provocan: insomnio, problemas de concentración, dolores de cabeza, presbicia precoz, entre muchos otros. Por otro, rebajan el proceso de lectura comprensiva requerido tanto para la academia como para interpretar cosas del mundo, a una mera lectura hipertextual, cuyas características tanto cognitivas como pragmáticas son radicalmente, distintas a las llevadas a cabo en la lectura en papel. Decir, como plantean algunos (Martínez, 2015; Da Pieve, 2016) que los jóvenes no leen menos, porque leen en otros espacios, otras cosas, y no las que se leían antes, lejos de una real apología de la juventud se acerca más a la justificación dada por una generación que no supo dotar de competencias de lecto-escritura necesarias a sus jóvenes.

Si bien es cierto que no se encontraron estudios específicos por fuera del mencionado que midan específicamente, la disponibilidad léxica, los indicios relevados permiten hipotetizar que, con el pasar de los años, los estudiantes llegan a los estudios superiores con un nivel de vocabulario menor. Es a raíz de esta hipótesis que se propuso este trabajo longitudinal, donde se analizaron los niveles de vocabulario de cuatro cohortes de ingresantes a distintas carreras universitarias en dos universidades nacionales, y se compararon las variaciones, así como correlaciones a efectos de observar cuán notable es la repercusión de los cambios de hábitos de lectura en el nivel de disponibilidad léxica productiva. 


\section{Metodología}

La investigación constó de dos instancias. En la primera se recaudaron datos de capacidad léxica productiva para cuatro cohortes de ingresantes a diferentes carreras de nivel superior, en cuatro años distintos (2015-2018). Luego se compararon los datos entre sí, empleando métodos descriptivos e inferenciales a efectos de determinar si los cambios en las puntuaciones habían resultado significativos o al menos relevantes.

\subsection{Instrumentos y materiales}

Se utilizó la prueba de vocabulario productivo Z-Test de sinónimos, para cuya elaboración se tomaron las Normas de Producción de Atributos Semánticos en Castellano Rioplatense (Vivas, Comesaña, García Coni, Vivas y Yerro, 2013), a partir de las cuales se obtuvieron 100 palabras de alta frecuencia que poseen sinónimos, según WordReference diccionario de sinónimos (2016). Luego, a través de la comparación con la base CREA de las 1000 palabras más frecuentes de la Real Academia Española (2016), y el juicio de pares especialistas en el área, empleando el método de agregados individuales (5 expertos), se llegó a una lista final de 40 palabras, de la que solo se tuvieron en cuenta el $100 \%$ de coincidencia favorable, para una mayor solidez del instrumento. Una vez obtenidos estos 40 ítems, se realizó una prueba de IVC con un valor de $.99, \mathrm{~N}=5 \mathrm{y} \mathrm{Ne}=5$, al ser expertos diferentes a los empleados en la etapa anterior del proceso y obtener una puntuación de 1 .

Para la validación externa, se realizó una prueba piloto $\mathrm{N}=32$, en la que se contrastaron los resultados obtenidos en el Z-Test con los derivados de evaluaciones formales del sistema de educación. Dado que hay un gran consenso entre la relación del vocabulario de amplio espectro con el desempeño académico, puesto que en este se ven involucradas tareas de lectura y escritura, se esperaba observar correlaciones entre ambos resultados. El resultado de la prueba ANOVA, con un p. crítico $=0.05$ y $\mathrm{F}=2.63$, fue de $\mathrm{p}=0.04$, mostrando una relación sólida entre la competencia léxica y el desempeño académico.

Para el cálculo del coeficiente de validez interna se contrastaron las puntuaciones obtenidas en la prueba piloto $\mathrm{N}=124$ con las logradas por los mismos sujetos en el test de denominación de Boston (Serrano et al. 2001). Aplicando una estadística de correlación lineal de Pearson, se obtuvo $r=0.78$, que es significativamente alto, si se considera que el test mencionado mide el vocabulario en sus dos dimensiones. Si a esto 
le sumamos la controversia histórica y actual sobre la necesidad o no de dividir dichas dimensiones, nos encontramos ante una validez interna que es al menos suficiente.

Dada la naturaleza de lo que se mide, el vocabulario, se descartó la posibilidad de utilizar un método de test-retest, puesto que había alto riesgo de que los sujetos desarrollasen aprendizaje. Por ello, se aplicó la técnica conocida como formas paralelas, en la que se emplearon dos versiones equivalentes del Z-Test, con una distancia de una hora entre ambas; se encontró un $r$ Pearson $=0.86$, lo cual indica que prácticamente, el 90 $\%$ de la varianza del test no se debe al error. En este test, las puntuaciones posibles pueden ir desde 0 a 40 y se delimitan 4 grupos de forma orientativa: A ( $>30$ alta competencia léxica), B $(20<30$ buena competencia léxica), $\mathrm{C}(10<20$ regular competencia léxica) y $\mathrm{D}(<10$ baja competencia). Además, para el procesamiento y análisis de los datos se emplearon los software End Note y el Statistic Package for Social Sciences (SPSS).

\subsection{Muestra}

El muestreo empleado fue probabilístico, usando una técnica de aleatorio simple entre la población de ingresantes a carreras de ciencias humanas de la Universidad Nacional de Mar del Plata (UNMDP) y de la Universidad Nacional de San Luis (UNSL). Se obtuvo N=318 estudiantes de primer año de carreras de humanidades; con un 59,74 $\%$ mujeres (190) y 41,26\% hombres (128); distribuidos a lo largo de cuatro años en el período $2015-2018$, a saber: $\mathrm{N} 1=83, \mathrm{~N} 2=68, \mathrm{~N} 3=79 \mathrm{y}$ N4=88; de entre 18 y 30 años $(x=19,5 ; \mathrm{s}=2,78 ; \mathrm{s} 2=5,62)$.

\subsection{Procedimiento}

Una vez obtenido el consentimiento oral que fue posteriormente, respaldado por su versión escrita, se procedió a la administración del Z-Test. Este se llevó a cabo de manera individual en el espacio de trabajo del docente-investigador, bajo condiciones óptimas de iluminación y sonido, sin distractor alguno. El tiempo de administración fue de 30 minutos, aunque ningún sujeto superó dicha medida. Este mismo procedimiento fue realizado un total de cuatro veces, a principios de los ciclos lectivos de los años 2015, 2016, 2017 y 2018. 


\subsection{Análisis}

Una vez obtenidas las puntuaciones de los test, se realizaron las estadísticas descriptivas de tendencia central (media, mediana, moda) junto con sus correspondientes medidas de dispersión (desviación típica, estándar y varianza), para cada una de las cohortes, en las cuales se analizaron correlaciones con la edad y el sexo. Asimismo, se aplicaron pruebas de normalidad para determinar las características de las puntuaciones obtenidas. Luego se comparó interanualmente, las puntuaciones obtenidas, para lo cual se analizó el coeficiente de variación de las puntuaciones, se estableció la función matemática para dicho coeficiente y se realizó un análisis de varianza (ANOVA de una cola), para determinar si había correlaciones estadísticamente significativas entre el año y las puntuaciones obtenidas en el test. Adicionalmente, se analizaron posibles incidencias de otras variables en las puntuaciones como el sexo y la edad.

\section{Resultados y discusión}

En primer lugar, vamos a analizar la distribución de las puntuaciones para cuatro años, observando la descripción general y los supuestos de normalidad.

Tabla 1. Descriptivos

\begin{tabular}{|c|c|c|c|c|}
\hline & & & Estadístico & $\begin{array}{c}\text { Error } \\
\text { estándar }\end{array}$ \\
\hline Puntuacion & media & & 21,7284 & ,42339 \\
\hline & $\begin{array}{l}95 \% \text { de intervalo de } \\
\text { confianza para la media }\end{array}$ & $\begin{array}{l}\text { Límite } \\
\text { inferior }\end{array}$ & 20,8954 & \\
\hline & & $\begin{array}{l}\text { Límite } \\
\text { superior }\end{array}$ & 22,5615 & \\
\hline & Media recortada al $5 \%$ & & 21,5092 & \\
\hline & Mediana & & 21,0000 & \\
\hline & Varianza & & 56,109 & \\
\hline & Desviación estándar & & 7,49057 & \\
\hline & Mínimo & & 7,00 & \\
\hline & Máximo & & 40,00 & \\
\hline & Rango & & 33,00 & \\
\hline & Rango intercuartil & & 10,00 & \\
\hline & Asimetría & & ,374 & 138 \\
\hline & Curtosis & &,- 260 & 275 \\
\hline
\end{tabular}

Nota: datos de los autores 
Lo primero que se observa es una puntuación media, para la totalidad de la muestra de 21,72 que permite afirmar que casi la totalidad de las puntuaciones se distribuye alrededor del centro de los valores. Esto es consistente con estudios anteriores (Zapico, 2018; Zapico y Zapico, 2018) en los que las puntuaciones relevadas para los primeros años tendían a situarse en 20 , que corresponde a un desempeño medio en el test. La mediana es consistente con este dato, situándose en 21. Esta idea se ve reforzada por las medidas de dispersión, que si bien muestran un rango amplio (33, por lo que hay puntuaciones desde 7 a 40), no resultan más que casos particulares, pues la desviación estándar de 7 es relativamente baja, al tratarse de una muestra amplia con valores de tendencia central consolidados en la puntuación media. Esto indica que, más allá del año de pertenencia, las puntuaciones tienden a ser similares. No obstante, también significa que hay una concentración de puntuaciones significativa por arriba y abajo de ese valor medio, que, si bien es de corto rango, en términos de vocabulario puede significar la diferencia entre un desempeño aceptable y uno no aceptable.

A este respecto Zapico y Delsouc (2017), establecieron el punto 25 como medida de puntuación necesaria para un desempeño académico aceptable, lo cual es consistente con lo que muchos autores señalan respecto a una baja relativa en las capacidades léxicas de los jóvenes en comparación con décadas anteriores. Esto es, ciertamente, algo llamativo y preocupante, dado que se esperaría que después de una formación primaria y secundaria se pudiera alcanzar fácilmente, la puntuación del punto de corte. Esto se vuelve particularmente cierto, al observar la asimetría, que adquiere un valor positivo alto lo que indica que, de esa masa de puntuaciones cercana a la media, una gran cantidad se encuentra por encima de ella, pero no lo suficiente como para alterar el valor de las medidas de tendencia central.

Luego, la curtosis nos habla de una formación platicurtica $(\mathrm{g} 2<0)$ que refuerza la idea de que hay gran cantidad de puntuaciones rondando el valor medio, pero alejadas levemente de él. Todos los datos analizados nos hablan de una muestra que, si bien tiende a consolidar puntuaciones medias en el test, no llegan a los valores esperados que correlacionan dichas puntuaciones con un desempeño académico al menos aceptable.

Finalmente, y pese a los niveles aparentemente altos de asimetría y curtosis, los estadísticos de normalidad, dan como resultado una distribución normal, como se observa en la tabla 2 y la figura 1. 
Tabla 2. Pruebas de normalidad

\begin{tabular}{|l|c|c|c|c|c|c|}
\hline & \multicolumn{3}{|c|}{ Kolmogorov-Smirnov ${ }^{\mathrm{a}}$} & \multicolumn{3}{c|}{ Shapiro-Wilk } \\
\hline & Estadístico & gl & Sig. & Estadístico & Gl & Sig. \\
\hline Puntuación &, 074 & 313 &, 000 &, 978 & 313 &, 000 \\
\hline
\end{tabular}

a. Corrección de significación de Lilliefors

Nota: datos de los autores

Ambas estadísticas confirman el supuesto de normalidad, que puede apreciarse en el siguiente gráfico Q-Q, el cual muestra un nivel de ajuste observado alto.

Figura 1

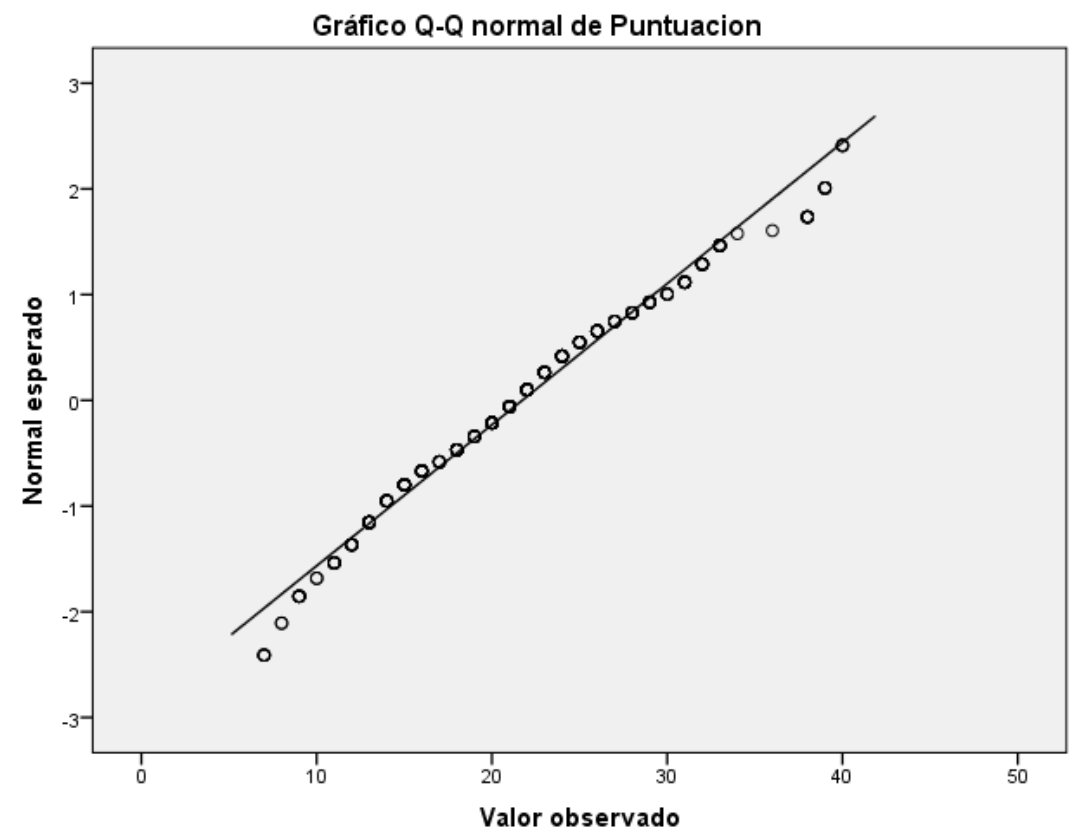

Notas: datos de los autores

Una vez establecida la normalidad de la muestra, se procedió a realizar las medidas descriptivas para las puntuaciones, pero discriminadas por años. 
Tabla 3. Comparación de medias por año

\begin{tabular}{|c|c|c|c|c|c|c|c|c|}
\hline Año & $\mathbf{N}$ & Media & $\begin{array}{c}\text { Desviación } \\
\text { estándar }\end{array}$ & $\begin{array}{c}\text { Error } \\
\text { estándar }\end{array}$ & $\begin{array}{c}\text { 95 \% del intervalo } \\
\text { de confianza para la } \\
\text { media }\end{array}$ & Mínimo & Máximo \\
\hline & & & & & $\begin{array}{c}\text { Límite } \\
\text { inferior }\end{array}$ & $\begin{array}{c}\text { Límite } \\
\text { superior }\end{array}$ & & \\
\hline 2015 & 83 & 24,0241 & 7,48816 &, 82193 & 22,3890 & 25,6592 & 7,00 & 40,00 \\
\hline 2016 & 63 & 22,0635 & 7,96539 & 1,00355 & 20,0574 & 24,0696 & 9,00 & 40,00 \\
\hline 2017 & 79 & 20,8354 & 7,46518 &, 83990 & 19,1633 & 22,5076 & 7,00 & 40,00 \\
\hline 2018 & 88 & 20,1250 & 6,68987 &, 71314 & 18,7076 & 21,5424 & 7,00 & 33,00 \\
\hline Total & 313 & 21,7284 & 7,49057 &, 42339 & 20,8954 & 22,5615 & 7,00 & 40,00 \\
\hline
\end{tabular}

Nota: datos de los autores

A simple vista encontramos que, con el pasar de los años, hay un descenso preocupante en las puntuaciones medias obtenidas. Las puntuaciones medias, que en primera instancia pareciera bajan poco (cuatro puntos nominales desde el 2015 hasta el 2018), representan un descenso equivalente al $10 \%$ del desempeño promedio entre el primer y el último grupo. Esto resulta en extremo llamativo, pues significa que, en cuatro años, hubo una pérdida promedio de las capacidades de vocabulario léxico a una velocidad constante, y que parece haberse estancado en el último año. A su vez dicha diferencia de medias no puede ser explicada por las desviaciones estándares, pues sus valores se mantuvieron constantes.

Estos resultados cuestionan directamente con todos los autores que afirman que los jóvenes no leen menos o tienen menos vocabulario, sino que se supone tienen otro vocabulario o leen otros textos. Si bien, ese enunciado quizá es verdadero en sí mismo, como investigadores de la educación que buscan, de acuerdo con lineamientos nacionales e internacionales, garantizar no solo el ingreso, sino también la permanencia y el egreso de los jóvenes en el nivel superior, la preocupación debería estar puesta en el léxico académico o en las capacidades de interpretación y producción léxica.

Por otro lado, estos números son consistentes con los planteos citados al principio de este artículo, que hablan de una disminución significativa en prácticas de lectura o empleo de medios analógicos para la comunicación. Esa disminución y cambio de hábito en la población joven, se refleja cada vez más en las puntuaciones obtenidas en este tipo de test o de similar desempeño (por poner un ejemplo, las pruebas estandarizadas para conocimientos de lengua y matemática). Incluso se 
puede realizar una observación teniendo en cuenta los valores mínimos y máximos observados en cada cohorte. En todas siempre hubo al menos un sujeto que logró la puntuación máxima en el test, y puntuaciones máximas subsecuentes para abajo con distintas frecuencias. Pero en la última cohorte medida, el valor más alto obtenido es de 33, lo que significa que ningún sujeto de dicho grupo obtuvo una puntuación más alta que esa. Esta disminución en el valor máximo puede parecer anecdótica, pero si se la combina con la disminución casi lineal año a año de los límites establecidos para valores inferiores y superiores, lo que ha sucedido es que ha bajado el rango promedio de los valores obtenidos. Las puntuaciones altas en media son cada vez más bajas, y las puntuaciones bajas en media, también son cada vez más bajas.

Continuando con el análisis, es interesante observar cómo la disminución está marcada fuertemente en los dos primeros años, pero en los dos últimos la baja se sostiene y ancla sobre una media de 20 puntos, que es la mitad de la escala que mide el test. Esto se refleja de manera más nítida en la tabla Tukey que hace comparaciones múltiples para los ANOVA, pero establecido año por año.

\section{Tabla 4. Comparaciones múltiples para ANOVA}

Variable dependiente: puntuación HSD Tukey

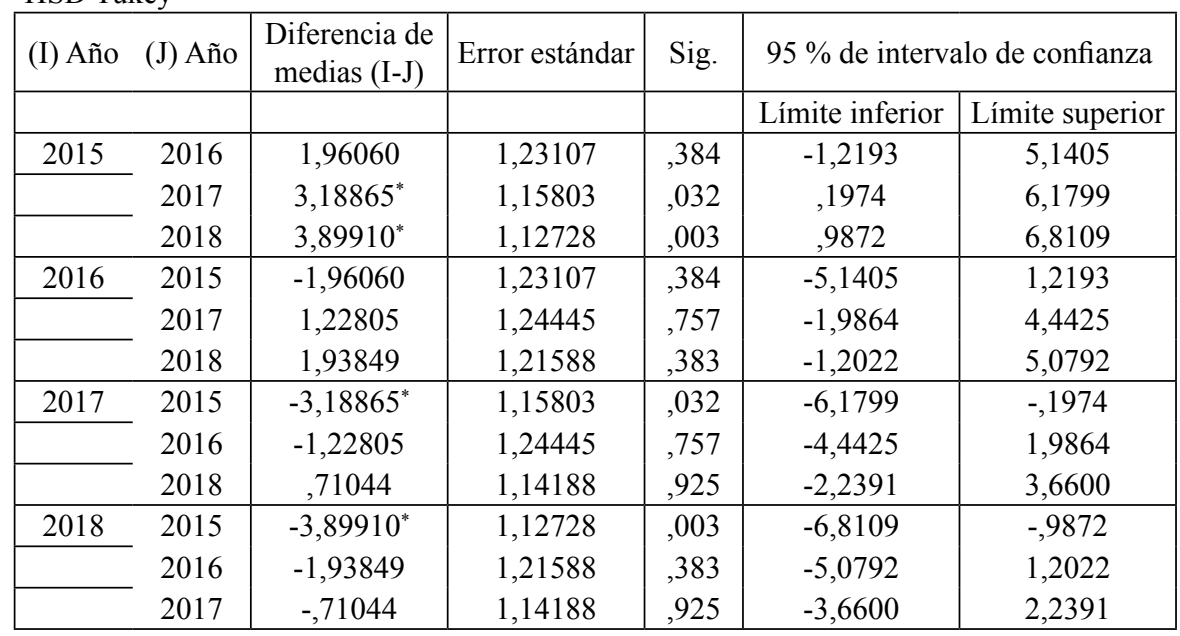

Nota: datos de los autores 
El año que se establece como punto de comparación central para nuestro análisis es el 2015, puesto que es el más alejado en el tiempo y el más susceptible para variar las puntuaciones. Nótese que, en el transcurso de un año, la diferencia entre las puntuaciones no es estadísticamente significativa, lo que significa que, aunque bajan, no lo hacen en gran medida. Sin embargo, la diferencia se acentúa estadísticamente hablando contra el año 2017 y aún mucho más contra el 2018. Este nivel de significancia estadística no se vuelve a repetir si se toma como punto de partida los próximos años, ni siquiera para el par 2016-2018, donde la diferencia de puntuaciones dista mucho de ser significativa. Esto requeriría una explicación, que en primera instancia podría atribuirse a una diferencia en la igualdad de medias para los grupos dado sus tamaños distintos, que a su vez podría incidir en la diferencia total.

No obstante, la Prueba Welch $(\mathrm{F}=4,63$; p.valor $=0,004)$, indica que la igualdad de medias es homogénea pese a los distintos tamaños de cada una de las muestras. Entonces, ¿Por qué, si bien la tendencia a la baja existe, hay un salto cuantitativo tan notable en el par 2015-2017 y no en otros pares de igual distancia? La respuesta parece estar dada por la homogeneidad de las varianzas, a partir del Estadístico de Levene ( $\mathrm{V}=0,31$; $\mathrm{p}$.valor $=0,81$ ), lo que significa que las varianzas involucradas en cada uno de los años no son iguales. Si nos remitimos puntualmente, a la varianza obtenida para cada uno de los años, encontramos una varianza bastante mayor en el año 2016 (Var=63,44), la más alta de los cuatro, junto para la muestra ( $\mathrm{N}=63$ ) más baja, lo cual implica que ha habido casos de puntuaciones más dispersas de lo normal. Es decir, de acuerdo con la tendencia esperada, las puntuaciones del año 2016 son altas, lo suficiente como para aproximarse al año 2015 , pero no lo suficiente como para marcar una diferencia significativa con los otros dos años por separado. Pese a esta anomalía, el análisis de varianza global muestra una aparente correlación entre el año y las puntuaciones. 
Tabla 5. ANOVA

\begin{tabular}{|ll|c|c|c|c|c|}
\hline & & $\begin{array}{c}\text { Suma de } \\
\text { cuadrados }\end{array}$ & gl & $\begin{array}{c}\text { Media } \\
\text { cuadrática }\end{array}$ & F & Sig. \\
\hline $\begin{array}{l}\text { Puntuación } \\
\text { Año }\end{array}$ & Entre grupos (Combinado) & 733,733 & 3 & 244,578 & 4,506 &, 004 \\
\hline & & 703,640 & 1 & 703,640 & 12,963 &, 000 \\
\hline & $\begin{array}{l}\text { Linealidad } \\
\text { Desviación de } \\
\text { la linealidad }\end{array}$ & 30,093 & 2 & 15,046 &, 277 &, 758 \\
\hline & 16772,184 & 309 & 54,279 & & \\
\hline Dentro de grupos & 17505,92 & 312 & & & \\
\hline
\end{tabular}

Nota: datos de los autores

Resulta útil la confirmación respecto a la importancia de año para determinar las puntuaciones en el test, el valor obtenido en el análisis de varianza. Puesto que, pese al caso anómalo ya destacado y explicado, el p.valor es suficiente para determinar un grado de asociación entre ambas variables. Aún más esclarecedor resulta el análisis de los estadísticos de asociación, específicamente eta $=0,2$ y eta $2=0,04$ que perfilan a la variable año como explicativa en buena medida de la variabilidad en la varianza, confirmando su significancia estadística, pero dejan abierta la posibilidad a la injerencia de otras variables que no se están analizando en este caso.

Una variable que podía estar incidiendo sería el sexo, pues está documentado con amplia historia las mayores capacidades léxicas en promedio que las mujeres suelen tener por encima de sus pares hombres. Con miras a esto, se analizaron también las medias comparadas por sexo.

Tabla 6. Puntuaciones medias por sexo

\begin{tabular}{|l|c|c|c|}
\hline Sexo & Media & N & $\begin{array}{c}\text { Desviación } \\
\text { estándar }\end{array}$ \\
\hline $\mathrm{H}$ & 21,3040 & 125 & 7,83419 \\
$\mathrm{M}$ & 22,0106 & 188 & 7,26061 \\
Total & 21,7284 & 313 & 7,49057 \\
\hline
\end{tabular}

Nota: datos de los autores

Una rápida revisión a las medias, observando que las desviaciones estándar son similares, evidencian lo esperado, una leve mejora de las puntuaciones en la población de mujeres. Sin embargo, esa leve 
diferencia no es explicativa de la diferencia de puntuaciones en prácticamente ninguna medida, simplemente, por el hecho de que, si la superioridad media existe en la muestra total, también existe en cada una de las cohortes. Algo diferente podría suponerse para la edad, puesto que si la edad no fuera homogénea en los grupos y a la vez dicha variable fuera altamente explicativa de la variedad de puntuaciones, una distribución no normal de la edad en alguno de los grupos podría haber tenido una incidencia al menos parcial en la curva de tendencia. A continuación, se presenta la tabla de puntuaciones medias obtenidas de acuerdo a la edad.

Tabla 7. Puntuaciones medias por edad

\begin{tabular}{|c|c|c|c|}
\hline Edad & Media & $\mathbf{N}$ & Desviación estándar \\
\hline 18 & 21,9481 & 135 & 7,59057 \\
19 & 19,7556 & 45 & 6,43554 \\
20 & 22,3448 & 29 & 7,50369 \\
21 & 24,2609 & 23 & 7,92704 \\
22 & 18,5556 & 9 & 5,87603 \\
23 & 22,3000 & 10 & 3,83116 \\
24 & 22,0000 & 4 & 4,00000 \\
25 & 23,6250 & 8 & 5,80486 \\
26 & 23,0000 & 9 & 6,92820 \\
27 & 15,0000 & 7 & 8,92562 \\
28 & 19,0714 & 14 & 7,31136 \\
29 & 23,9091 & 11 & 9,44939 \\
30 & 26,0000 & 9 & 9,69536 \\
Total & 21,7284 & 313 & 7,49057 \\
\hline
\end{tabular}

Nota: datos de los autores

Es curioso observar que, a simple vista, pareciera haber una relación positiva entre las variables edad y puntuaciones. Ciertamente, no es una relación de naturaleza lineal, pero las puntuaciones de léxico parecieran subir un poco a medida que aumenta la edad. Esta intuición podría ser validada por el prejuicio de que a mayor edad mayor formación, pero eso no es necesariamente cierto. Para confirmar o desmentir esta hipótesis, se muestra a continuación un modelo de regresión lineal para las dos variables (año y edad), donde se observa la incidencia que cada una tiene sobre las puntuaciones a partir de las definiciones de las pendientes y la ordenada al origen para cada recta hipotética. 
Tabla 8. Coeficientes ${ }^{\mathrm{a}}$

\begin{tabular}{|c|c|c|c|c|c|}
\hline Modelo & \multicolumn{2}{|c|}{$\begin{array}{c}\text { Coeficientes no } \\
\text { estandarizados }\end{array}$} & $\begin{array}{c}\text { Coeficientes } \\
\text { estandarizados }\end{array}$ & $\mathrm{T}$ & Sig. \\
\hline & $\mathrm{B}$ & Error estándar & Beta & & \\
\hline 1 (Constante) & 2658,194 & 725,722 & & 3,663 &, 000 \\
\hline Edad &, 066 &, 114 &, 032 &, 580 &, 562 \\
\hline Año & $-1,308$ &, 360 &,- 202 & $-3,634$ &, 000 \\
\hline
\end{tabular}

a. Variable dependiente: puntuación

Nota: datos de los autores

Como se observaba intuitivamente en la tabla general, hay un incremento leve de unidad, a partir de la variable edad (una pendiente de 0,66$)$, pero dicha relación de cambio tiene poco o nada de poder explicativo en el modelo combinado con la variable año, que por su parte tiene una pendiente de $-1,30$ unidades y un nivel de significancia estadística plena.

\section{Conclusión}

A lo largo del texto, hemos analizado las posibles relaciones entre el pasar del tiempo, concretamente cuatro años, y las puntuaciones obtenidas en un test de vocabulario productivo. Naturalmente, nos encontramos ante un asunto muy complejo por la gran cantidad de potenciales variables no estudiadas y que podrían en alguna medida explicar los resultados obtenidos.

En primer lugar, hay que destacar lo que se ha podido constatar, que existe una relación entre el paso del tiempo y la disminución en las puntuaciones obtenidas en el mismo test. Esto nos habla, precisamente, de un fenómeno que debe preocupar a cualquier investigador de educación: la cada vez menor riqueza léxica con la cual los jóvenes afrontan sus carreras de nivel superior. Esta menor riqueza no solo preocupa, en tanto presupone una mayor dificultad para resolver las mismas situaciones académicas que años atrás se hacían más fácilmente, sino también una disminución en las capacidades comunicativas de las generaciones venideras. Recordemos que el lenguaje no es solo una herramienta de resolución de problemas, es un elemento necesario para la comunicación, la expresión de emociones, la posibilidad de vincularnos con otros, es un elemento estructurante en nuestro tejido social. 
En segundo lugar, se han analizado someramente, la posible incidencia de otras variables como sexo y edad. Para sexo se ha determinado lo que otros estudios ya afirmaban, una leve mejoría del desempeño en habilidades lingüísticas por parte de las mujeres, pero nada significativo estadísticamente, o al menos con poder explicativo para este estudio. Por otro lado, la variable edad resulta interesante, en tanto muestra algún nivel de asociación, pero sin ninguna direccionalidad particular, no se puede buscar relación alguna entre las puntuaciones obtenidas y la edad. Una hipótesis posible sería pensar, con base en nuestros resultados, que las personas más adultas tenderán a tener un desempeño mayor, por el hecho de que en cohortes anteriores se han tenido mejores resultados que en posteriores, sin embargo esto no es válido porque a la larga, por exposición académica, se adquirirá un mejor desempeño y las puntuaciones más bajas se nivelarán con las más altas, como se observó en un estudio longitudinal anterior (Zapico, 2018).

Más allá de preguntarnos por qué, gesto que es válido y necesario en la medida que se busque dar una solución al problema, es preciso concientizarnos del papel vital que el vocabulario adquiere a la hora de entender las problemáticas vinculadas al ingreso, permanencia y egreso de los alumnos en el nivel superior. Esta concientización, además, debe venir de la mano de políticas concretas de intervención temprana, tanto en el nivel primario y secundario, adaptadas a una realidad social y tecnológica más compleja. Las formas de lectura y acceso al conocimiento han cambiado, por ende, nuestra responsabilidad para con el léxico debe cambiar también. No es cuestión de limitarse a decir que los jóvenes realizan actividades diferentes a las que hacíamos nosotros, puesto que, si bien es cierto que las hacen, es nuestro deber lograr el máximo nivel de desarrollo léxico posible en ellos, para brindarles no solo una mejor preparación académica, sino también humana, para un mundo cada vez más complejo.

\section{Referencias}

Álvarez, G. U., y Martínez, Z. C. (2011). Prácticas de lectura y escritura académicas en la universidad colombiana. Magis, Revista Internacional de Investigación en Educación, 3(6), 317-341.

Amiama, C., y Mayor, C. (2017). Lectura digital en la competencia lectora: la influencia en la Generación Z de la República Dominicana. Comunicar, 52(XXV), 105-114. 
Armengol, A. R. M., Castellanos, K. M., Molina, M. M., León, Á. M. O. y Díaz, A. G. (2017). Factores de riesgo para enfermedades oculares. Importancia de la prevención. Revista Finlay, 7(1), 153-161.

Belaunde, D. H. M., Carrillo, I. A., Amo, Á. y Ayora, M. (2016). Relación entre trabajo con pantalla de visualización de datos (PVD) y aumento de la presión intraocular (PIO) en trabajadores en Málaga, Andalucía. ORPjournal, 5(1), 3-14.

Bojorque, G., Bojorque, V. y Dávalos, J. (2016). Variables personales relacionadas con el rendimiento académico. Madrid: Apeiron.

Carlino, P. (2007). ¿Qué nos dicen las investigaciones internacionales sobre escritura en la universidad? Cuadernos de Psicopedagogía, 4(1), 21-40.

Carlino, P. (2001). Hacerse cargo de la lectura y la escritura en la enseñanza universitaria de las ciencias sociales y humanas. Ponencia presentada en las Jornadas sobre la lectura y la escritura como prácticas académicas universitarias, Universidad Nacional de Luján.

Da Pieve, E. (2016). ¿Los jóvenes leen menos que antes? Buenos Aires: Letras.

Díaz, A. A., Reyes, N. B. y Rangel, L. E. C. (2017). Efectos de los dispositivos electrónicos sobre el sistema visual. Revista Mexicana de Oftalmología, 91(2), 103-106.

Garfias, E. (2010). Los jóvenes no leen menos. Periódico La Jornada, Domingo 6 de junio de 2010. Recuperado de http://www. jornada.unam.mx/2010/06/06/cultura/a05n1 cul

Hernández, B., García, C. G. y López, C. I. (2015). La lectura y la escritura a través de la cibernética social: herramientas creadoras de mundo para los jóvenes. Infancias Imágenes, 14(2), 71-88.

Hirsch, E. D. (2004). La comprensión lectora requiere conocimiento de vocabulario y del mundo. Estudios Públicos, 108(1), 229-252.

Horacek, G. A. E., Ottaviano, M. L. y Palacios, N. G. (2015). Migraciones, digitalizaciones y prácticas educativas en el contexto argentino. Educatio Siglo XXI, 33(3), 63-84.

Laco, L., Natalia, L. y Ávila, M. (2010). La lectura y escritura en la formación académica, docente y profesional. Buenos Aires: Editorial de la Universidad Tecnológica Nacional. 
Ledo, M. V. (2016). Síndrome del Ordenador. Revista INFODIR, 6(1), $1-4$.

León, F. R. (2015). The east-to-west decay of math and reading scores in the United States: A prediction from UVB radiation theory. Personality and Individual Differences, 86(1), 287-290.

Londoño-Vásquez, D. A. y Bermúdez, H. L. (2018). Literacy levels in University Students: Qualitative Interviews and Sociolinguistic Analysis. Revista Latinoamericana de Ciencias Sociales, Niñez y Juventud, 16(1), 315-330.

Martínez, S. L. (2015). Los jóvenes, las tecnologías y la escuela. De tecnologías digitales, educación formal y políticas públicas, 27(1), 14-27.

Martínez, C. R. (2017). ¿Qué pasa entre los jóvenes y las revistas?: Un vistazo al consumo de revistas de los consumidores de entre 14 y 34 años. Anuncios: Semanario de Publicidad y Marketing, 1558, 36-37.

Moyano, E. (2005). La escritura académica: una tarea interdisciplinaria a lo largo de la currícula universitaria. Textura, 4(4), 109-120.

Mumper, M. L. y Gerrig, R. J. (2017). Leisure reading and social cognition: A meta-analysis. Psychology of Aesthetics, Creativity, and the Arts, 11(1), 109-120.

Ontibón, E. (2018). Lectura crítica de ensayos en educación media. Madrid: Apeirón.

Paredes, J. (2015). La escuela y el desafío del hábito de la lectura. The school and the challenge of reading habit. Razón y Palabra, 19(1), 288-345.

Pavez, A., Rojas, P., Rojas, F. y Zambra, N. (2016). Habilidades semánticas y rendimiento académico en escolares de 2 y 4 año básico. Revista Chilena de Fonoaudiología, 15(1), 1-12.

Requejo, I. (2001). Pobreza de vocabulario y autoría de la palabra $y$ del pensamiento. Recuperado de http://usuarios.arnet.com.ar/ yanasu/educacion.htm

Roldán, L. A. y Zabaleta, V. (2016). Lectura y escritura. Autopercepción del desempeño en estudiantes universitarios. Revista Iberoamericana de Diagnóstico y Evaluación e Avalição Psicológica, RIDEP, 2(42), 27-38.

Sánchez, C. (2003). ¿Por qué los jóvenes leen mal? Madrid: Asociación Educación y Bibliotecas Tilde. 
Serrano, C., Allegri, R. F., Drake, M., Butman, J., Harris, P., Nagle, C. y Ranalli, C. (2001). Versión abreviada en español del test de denominación de Boston: su utilidad en el diagnóstico diferencial de la enfermedad de Alzheimer. Revista de neurología, 33(7), 624-627.

Valeiras, N. (2009). El rendimiento académico y la escritura en ingresantes de carreras de ciencias exactas. Revista Educación en Biología, 12(1), 15-27.

Zapico, G. (2018). Adquisición de vocabulario productivo en contextos académicos superiores: análisis de su evolución a partir de un estudio longitudinal. Revista Ensayos Pedagógicos, 13(2), 119-137.

Zapico, G. (2017). Ensayo sobre la necesidad de la enseñanza directa de vocabulario en los niveles primario y secundario del sistema educativo. Revista Ensayos Pedagógicos, 12(2), 33-45.

Zapico, G. (2016). Evaluación de desempeño académico: la competencia léxica productiva como competencia con validez predictiva. Revista Ensayos Pedagógicos, 11(2), 159-171.

Zapico, G. y Delsouc, A. (2017). Competencia léxico-productiva, memoria a corto plazo y desempeño académico en el nivel superior: correlaciones a partir de un análisis simple y uno multivariado. Revista Ensayos Pedagógicos, 12(1), 149-165.

Zapico, G. y Zapico, G. (2018). La competencia léxico-productiva como predictor del desempeño académico. Revista Ensayos Pedagógicos, 13(1), 223-243. 
